\title{
DESENVOLVIMENTO DE UM APLICATIVO WEB EDUCACONAL PARA AUXILIAR NO ENSINO REMOTO DE PROCESSAMENTO DIGITAL DE SINAIS
}

Adriel de Oliveira Freitas - adrieloliveira_@hotmail.com

Universidade da Integração Internacional da Lusofonia Afro-Brasileira - Campus Redenção $R$. José Franco de Oliveira, s/n-Zona Rural

CEP 62790-970 - Redenção - Ceará

João Paulo do Vale Madeiro - jpaulo.vale@dc.ufc.br

Universidade Federal do Ceará, Departamento de Computação

R. Cinco, 100 - Pici

CEP 60355-636-Fortaleza - Ceará

Marciel Barros Pereira-marciel@crateus.ufc.br

Universidade Federal do Ceará - Campus Crateús

Rodovia BR-226, Km 03, s/n

CEP 63700-000 - Crateús - Ceará

Alane Teixeira Rodrigues - alane.teixeira.r@gmail.com

Universidade Federal do Ceará - Campus Sobral

Rua Estenilau Frota, s $n$ - Centro

CEP 62010-560 - Sobral - Ceará

Vandilberto Pereira Pinto - vandilberto@yahoo.com.br

Universidade da Integração Internacional da Lusofonia Afro-Brasileira - Campus Redenção

$R$. José Franco de Oliveira, s/n-Zona Rural

CEP 62790-970 - Redenção - Ceará

Resumo: A pandemia do COVID-19 contribuiu para o cancelamento das aulas presenciais em universidades de todo o mundo. Como resultado, as instituições de ensino tiveram que remodelar radicalmente suas técnicas de ensino-aprendizagem, levando em consideração a utilização de ferramentas e plataformas de ensino remoto. Um dos principais problemas enfrentados por instituições públicas, consiste na inclusão digital dos alunos em situação de vulnerabilidade econômica. Esses alunos dificilmente possuem um computador com software licenciado em casa para aplicar conceitos de processamento digital de sinais sem depender do laboratório da instituição de ensino. Com base nessas premissas, o presente trabalho apresenta o desenvolvimento de um aplicativo web educacional para auxiliar no ensino remoto de processamento digital de sinais, a partir da utilização de um smartphone, computador ou tablet. O aplicativo funciona online, o aluno pode acessar remotamente através de um link. A partir dos resultados apresentados é possível observar que o aplicativo Web apresentou um bom desempenho tanto no acesso remoto, como na aplicação de técnicas de processamento de sinais.

Palavras-chave: Ensino Remoto, Aplicativo Web, Processamento de Sinais. 


\section{INTRODUÇÃ̃O}

Diante da pandemia causada pelo COVID - 19, as instituições de ensino enfrentaram o grande desafio de adaptar suas aulas presenciais a formas de ensino remota, com auxílios de ferramentas de vídeos chamadas online, bem como aplicativos de celulares e programas de computadores que facilitam a aprendizagem dos conteúdos ensinados. Segundo a UNESCO (2020), no dia 21 de abril de 2020, 90\% dos alunos do mundo estavam impossibilitados de frequentar as aulas presenciais. Em países como o México, Chile e Uruguai, houve iniciativas de uso de tecnologias digitais na educação, que envolvem aplicativos gratuitos, programas de televisão e plataformas de aprendizagem (ARRUDA, 2020, p 261). No Brasil, as escolas e universidades estão fazendo uso de plataformas como Zoom e Google Meet para facilitar a interação entre alunos e professores, de forma que as aulas aconteçam com uma boa comunicação entre discentes e docentes.

Nas universidades brasileiras, particularmente nos cursos de tecnologia, existem diversas disciplinas que dependem da utilização de laboratórios com computadores dotados de um hardware robusto e com softwares licenciados para simular o comportamento de dispositivos eletrônicos, simular sistemas complexos, e realizar análise de sinais. Com as aulas presenciais suspensas, é necessário que essas disciplinas sejam ministradas de forma remota e sem a utilização de laboratórios. Para que o impacto negativo na qualidade do ensino remoto dessas disciplinas venha ser minimizado, alguns professores estão utilizando aplicativos Web para realizar análise de sinais, simular sistemas complexos, e simular o comportamento de dispositivos eletrônicos. Com isso, os alunos podem ter acesso à recursos de simulação e análise de sinais sem precisar utilizar o laboratório da instituição. Esse acesso pode ser realizado a partir de um smartphone, tablet ou um computador (sem a necessidade de possuir um hardware robusto).

Algumas instituições de outros países, antes da pandemia do COVID-19, já utilizavam Aplicativos Web como ferramenta no processo de ensino e/ou aprendizado de conteúdos de disciplinas ministradas tanto de forma presencial, como remota. Por exemplo, Integrantes do departamento de engenharia elétrica da Ghent University desenvolveram, com o auxílio do Matlab App Designer, um Aplicativo Web para auxiliar os alunos no aprendizado de Sistemas Dinâmicos e Controle Online (ODYSC), esse aplicativo passou a ser utilizado nos cursos introdutórios de dinâmica e controle da Ghent University (DEKEMELE et al, 2018, p. 1). Outro Aplicativo Web foi desenvolvido por integrantes do departamento de eletrônica da Universidade de Sakarya, com o objetivo de facilitar o aprendizado de técnicas de modulação digital no curso de sistemas de comunicação. Também foi utilizado o MATLAB App Design para a criação de uma interface educacional web (BAYILMIS, 2010, p. 1).

Para facilitar o estudo sobre comunicações ópticas, Integrantes Instituto Nacional de Astrofísica Óptica e Eletrônica(localizado no México), desenvolveram um aplicativo para ser utilizado como ferramenta para calcular e plotar facilmente o espectro óptico de fontes ópticas de modo único e multimodo, bem como a resposta de frequência do filtro fotônico de microondas (MPF) usado em um sistema de comunicação óptica (FERNÁNDEZ, 2020, p.4).

Muitos dos programas utilizados em universidades para o ensino de Processamento Digital de Sinais, requerem licença de utilização e um computador com requisitos mínimos de hardware, por isso, é comum que os alunos só tenham acesso a esses programas nos computadores dos laboratórios de suas instituições de ensino. Com a pandemia causada pelo novo coronavirus (COVID - 19), nem todos os alunos poderiam ter acesso aos softwares necessários para darem continuidade aos estudos online. Com base nessas premissas, o presente 
trabalho propõe o desenvolvimento de um Aplicativo Web para auxiliar no ensino remoto de processamento digital de sinais. Para desenvolver o Aplicativo foi utilizado as ferramentas App Designer e MATLAB Web App Server do software MATLAB Compiler. O aplicativo proposto pode ser acessado de qualquer local do país, para isto, basta digitar um endereço URL na barra de pesquisa do navegador de um smartphone, tablet ou computador.

Assim, o artigo está organizado da seguinte forma. $\mathrm{Na}$ Seção 2 é apresentado o ambiente onde foi desenvolvido o Aplicativo Web proposto. Na Seção 3 é feita uma apresentação sobre a ferramenta utilizada para hospedar o aplicativo $\mathrm{Web}$, com isso permitir que o usuário possa acessá-lo de forma remota. Já na Seção 4 são apresentados os materiais utilizados no desenvolvimento do Aplicativo, além dos procedimentos para criar, hospedar, executar, e acessar remotamente o Aplicativo Web. Na Seção 5 são apresentados os resultados de testes feitos com o aplicativo, no quesito de acesso remoto e aplicação de técnicas de processamento. E finalmente, na Seção 6 é apresentada a conclusão e as perspectivas para trabalhos futuros.

\section{APP DESIGNER}

O App Designer consiste em um ambiente de programação do MATLAB Compiler, criado para fins de desenvolvimento e compartilhamento de aplicativos independentes (MATHWORKS, 2020a). Com o auxílio do App Designer é possível desenvolver aplicativos de alta qualidade apenas movendo os componentes visuais para definir o design da sua interface gráfica com o usuário (GUI) e utilizando o editor integrado para inserir algoritmos em MATLAB (MATHWORKS, 2020b). A Figura 1 ilustra o ambiente de desenvolvimento do App Designer.

Figura 1 - Ambiente de desenvolvimento do App

Designer.

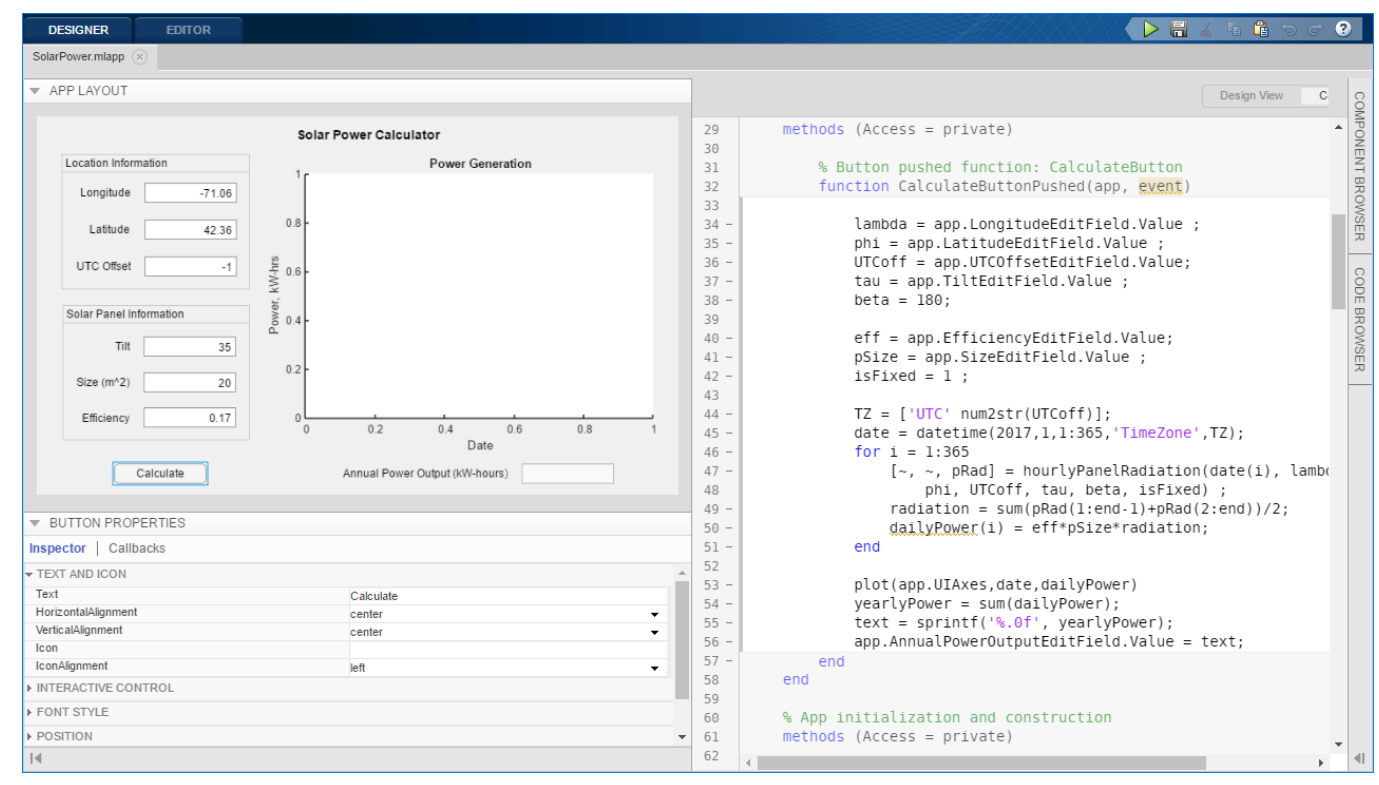

Fonte: (MATHWORKS, 2020b)

O App Designer pode ser utilizado para gerar dois tipos de aplicativos independentes: Aplicativo Executável e Aplicativo Web. Os Aplicativos Executáveis possuem um formato de extensão exe e podem ser executados em máquinas com sistema operacional Windows ou Linux 
(MATHWORKS, 2020b). Os Aplicativos executáveis podem ser compartilhados com usuários finais sem a aplicação de royalties. Para isto, é necessário que seja instalado o MATLAB Runtime na máquina que irá executar o Aplicativo Executável (MATHWORKS, 2020a). Já os Aplicativos Web possuem um formato de extensão $c t f$. Esse tipo de aplicativo necessita ser hospedado em um Servidor Web, ou em uma plataforma de armazenamento em nuvem (MATHWORKS, 2020c). Após serem hospedados, os aplicativos web são acessados remotamente através de um link, utilizando smartphones, tablets ou computadores.

O App Designer foi utilizado no presente trabalho para desenvolver o Aplicativo Web Educacional para fins de aplicação de técnicas de processamento de sinais remotamente.

\section{MATLAB WEB APP SERVER}

O MATLAB Web App Server consiste em um software desenvolvido com o objetivo de hospedar aplicativos do MATLAB como aplicativos interativos da web (MATHWORKS, 2020c). Para isto, é necessário que os aplicativos sejam desenvolvidos no MATLAB App Designer. Esse software pode ser utilizado em uma Unidade Central de Processamento (CPU), ou nas plataformas de armazenamento em nuvem AWS e Azure (JCMWAVE, 2020). Uma vez hospedados, os Aplicativos Web podem ser controlados de forma remota a partir do navegador de um smartphone, computador ou tablet. É importante que os aplicativos sejam desenvolvidos utilizando o recurso Auto-reflow, esse recurso permite que haja um ajuste automático do tamanho da janela de interação homem-máquina, em outras palavras, permite que o Aplicativo Web se adapte à resolução de tela de qualquer dispositivo (MATHWORKS, 2020e).

$\mathrm{O}$ acesso remoto aos aplicativos ocorre de três métodos distintos. O primeiro método consiste em um acesso local aos aplicativos hospedados em uma CPU, esse acesso só pode ser estabelecido por dispositivos conectados na mesma rede do CPU. O acesso local é indicado para instituições que desejam que os aplicativos WEB rodem apenas em redes internas, com isso, os aplicativos só irão funcionar dentro das dependências da instituição. O segundo método de acesso aos Aplicativos Web é denominado de acesso externo, nele os dispositivos conectados em qualquer rede exceto a rede do CPU que hospeda o Aplicativo. O acesso externo é indicado para instituições que desejam que os aplicativos WEB sejam utilizados como ferramenta de ensino remoto, permitindo assim, que alunos acessem os aplicativos de qualquer lugar do país. Já o terceiro método de acesso pode ser utilizado tanto por acesso local, como por acesso externo. Para isto, é necessário hospedar os Aplicativos Web em uma plataforma de armazenamento em nuvem. Vale ressaltar que a hospedagem de aplicativos em plataforma de armazenamento em nuvem gera um custo adicional, porém as plataformas AWS e Azure fornecem 1 ano de utilização gratuita.

O software MATLAB Web App Server foi utilizado no presente trabalho para hospedar o Aplicativo Web Educacional proposto neste artigo.

\section{MATERIAIS E MÉTODOS}

Nesta seção, são apresentados os materiais utilizados para criar um Aplicativo Web Educacional para auxiliar no ensino de processamento digital de sinais. Em seguida são apresentados os procedimentos realizados para criar, executar e acessar remotamente o 
aplicativo proposto. Os mesmos procedimentos podem ser executados para o desenvolvimento de Aplicativos Web para aplicações em diversas áreas da Engenharia.

\subsection{Materiais}

Para desenvolvimento do Aplicativo Web proposto, foi utilizado o software MATLAB Compiler, através dele é possível utilizar as ferramentas MATLAB App Designer e MATLAB Web App Server. Para utilizar o MATLAB Compiler é necessário possuir um computador com os seguintes requisitos mínimos: 4GB de memória RAM, Windows 10(Versão 1709 ou superior), qualquer processador Intel ou processador AMD x86-64, 31GB de memória de Disco, e uma placa gráfica acelerada por hardware que suporta OpenGL 3.3 com GPU de 1GB de memória(MATHWORKS, 2020d). Para criar o Aplicativo Web Educacional proposto, foi utilizado um notebook Dell de $8^{\circ}$ geração, 8GB de memória RAM, 1TB de memória de Disco, Processador Intel i7 e uma placa gráfica de $2 \mathrm{~GB}$ dedicada.

\subsection{Desenvolvimento e Execução do Aplicativo Web Educacional}

Utilizando o software MATLAB Compiler, foi desenvolvido um Aplicativo Web Educacional. Nele foram implementados algoritmos em MATLAB para agregar as seguintes técnicas de processamento digital de Sinais: filtro passa-alta, filtro passa-baixa, filtro passabaixa, filtro rejeita-faixa, e decomposição Wavelet multinível utilizando as famílias de funções: Daubechies, Biorthogonal, Coiflets, Symlets, Discrete Meyer, e Reverse Biorthogonal. A partir desse aplicativo, os professores que ministram disciplinas relacionadas à processamento digital de sinais podem demonstrar na prática a aplicação de filtros digitais em sinais, realizar uma análise espectral no domínio da frequência, além de realizar análise de sinais com base em decomposição Wavelet. Os alunos podem acessar remotamente o Aplicativo Web Educacional a partir de qualquer localidade do país. Os procedimentos realizados para criar, executar e acessar remotamente o Aplicativo Web Educacional foram divididos em 6 etapas, conforme detalhadas abaixo:

\section{Etapa 1: Instalar o software MATLAB Compiler}

Nesta etapa foi instalado software MATLAB Compiler em um computador com os requisitos mínimos exigidos. Vale ressaltar que vários recursos importantes do MATLAB Compiler estarão disponíveis apenas na versão 2017a ou superior.

\section{Etapa 2: Abrir o ambiente de desenvolvimento App designer}

Nesta etapa foi aberto o ambiente de desenvolvimento App designer, para começar a programar o Aplicativo Web Educacional.

\section{Etapa 3: Criar uma interface de usuário e escrever o código para o comportamento do Aplicativo Web Educacional}

Nesta etapa foi desenvolvido a interface gráfica de interação homem-máquina do Aplicativo Web Educacional, e foi inserido códigos em MATLAB para agregar as funcionalidades de processamento digital de sinais no aplicativo. É nesta etapa que o usuário deve definir a utilidade do aplicativo. Os algoritmos devem ser inseridos conforme a 
necessidade da aplicação, levando em consideração a área do conhecimento na qual o aplicativo será utilizado.

\section{Etapa 4: Compilar o aplicativo}

Após desenvolver a interface gráfica e inserir os algoritmos para comportamento do Aplicativo Web Educacional, é necessário compilar esse aplicativo. Para isto, basta clicar na opção "share/Web App". Após o término da compilação, foi gerado um arquivo com formato de extensão $c t f$. Esse arquivo gerado consiste no Aplicativo Web.

\section{Etapa 5: Hospedar o app web}

Nesta etapa foi realizado o processo de hospedagem do Aplicativo Web Educacional, foi utilizado um processo de hospedagem para fins de acesso remoto externo. Para hospedar um aplicativo para ser acessado remoto, utilizando uma rede local ou uma rede externa é necessário armazenar o arquivo ctf (gerado na etapa anterior) em uma pasta denominada App. A pasta App fica localizada no disco local do CPU, ela é criada automaticamente pela ferramenta MATLAB Web App Server. Já para hospedar um aplicativo utilizando uma plataforma de armazenamento em nuvem, é necessário realizar os seguintes procedimentos na plataforma AWS ou Azure: instalar uma máquina virtual, inserir MATLAB Web App Server e fazer o upload do arquivo ctf (gerado na etapa anterior).

\section{Etapa 6: Acessar remotamente o Aplicativo Web Educacional}

Após hospedar o Aplicativo Web Educacional, é possível acessar através do navegador de um dispositivo móvel. Para acessar o Aplicativo Web Educacional de forma remota, utilizando computadores, smartphone, ou tablets conectados à rede local, é necessário abrir o navegador do dispositivo e digitar a URL formada pelo IP local da CPU que hospeda o Aplicativo Web e o link do Aplicativo que deseja acessar. De modo análogo, para acessar o Aplicativo Web Educacional de forma remota, utilizando computadores, smartphone, ou tablets conectados em uma rede externa utilizando a internet, é necessário abrir o navegador do dispositivo e em seguida digitar a URL formada pelo IP externo da CPU que hospeda o Aplicativo Web e o link do Aplicativo que deseja acessar. No presente trabalho foi implementado um acesso externo, já que o aplicativo foi criado para auxiliar o professor em aulas remotas.

\section{RESULTADOS E DISCUSSÕES}

Após o processo de desenvolvimento e hospedagem do Aplicativo Web Educacional foram realizados testes para verificar o funcionamento do Aplicativo em situação de acesso remoto local e externo, além de testes para verificar o desempenho do aplicativo em situação de aplicação de técnicas de processamento digital de sinais.

Após a execução testes, foi possível constatar que o aplicativo Web Educacional apresentou um satisfatório desempenho, quando acessado e controlado remotamente, tanto por dispositivos móveis conectados na rede local como por dispositivos móveis conectados a uma rede externa. A Figura 2 a seguir mostra uma foto do Aplicativo Web Educacional sendo acessado remotamente: 
Figura 2 - Aplicativo Web Educacional sendo acessado remotamente através do navegador de um dispositivo móvel

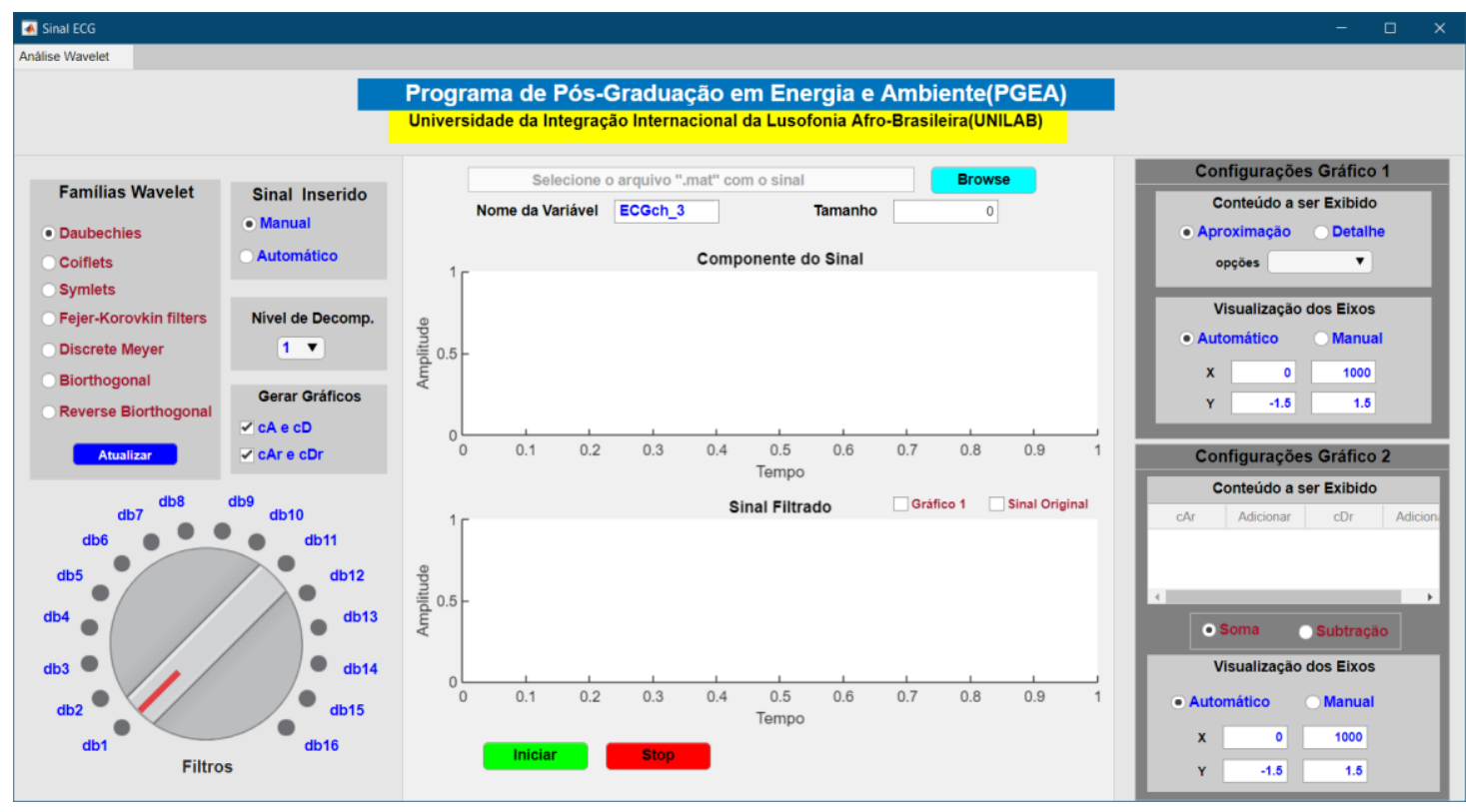

Fonte: Próprio Autor

A partir da Figura 2 é possível observar a interface de interação homem-máquina do Aplicativo Web Educacional desenvolvido no presente trabalho. A partir dessa interface é possível inserir um arquivo (de formato de extensão: txt, $\operatorname{csv}$, mat) contendo um sinal em sua forma discreta. Após inserir um sinal é possível aplicar técnicas de processamento digital para análise de sinais. O Aplicativo Web Educacional não apresentou falhas durante os testes de aplicação de técnicas de processamento digital de sinais, quando utilizado de forma remota.

Como teste, foi inserido um sinal de eletrocardiograma para aplicar alguns filtros e uma decomposição multinível Wavelet. A Figura 3 a seguir apresenta a utilização do Aplicativo para aplicar um filtro passa baixa com frequência de corte em 64Hz (Gráfico Superior) e um filtro passa faixa com banda passante de 0 à $16 \mathrm{~Hz}$ (Gráfico Inferior): 


\section{COBENGE e III Simpósio Internacional de Educaçāo em Engenharia da ABENGE}

\section{"Os desafios para formar hoje o engenheiro do amanhã"}

$\mathrm{Ol} \mathrm{a} \mathrm{O3}$ de dezembro Evento On-line

Figura 3 - Utilizando o Aplicativo Web Educacional para aplicar um filtro passa baixa com frequência de corte em $64 \mathrm{~Hz}$ (Gráfico superior) e um filtro passa faixa com banda passante de 0 à $16 \mathrm{~Hz}$ (Gráfico inferior)

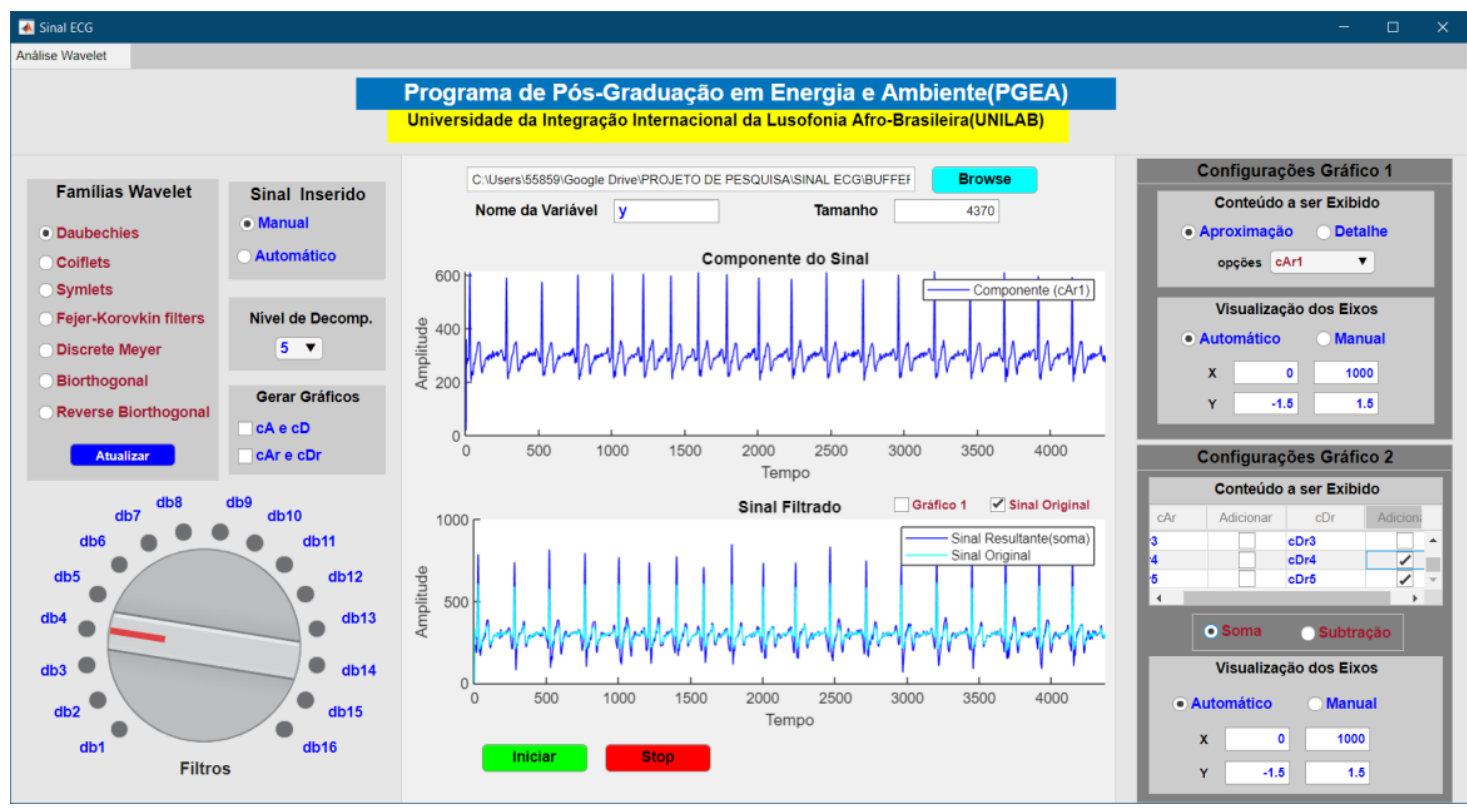

Fonte: Próprio Autor

A Figura 4 a seguir apresenta o resultado retornado pelo aplicativo após a aplicação de uma decomposição wavelet em 4 níveis, utilizando Daubechies $(d B 4)$ :

Figura 4 - Decomposição Wavelet em 4 níveis, utilizando Daubechies (dB4)

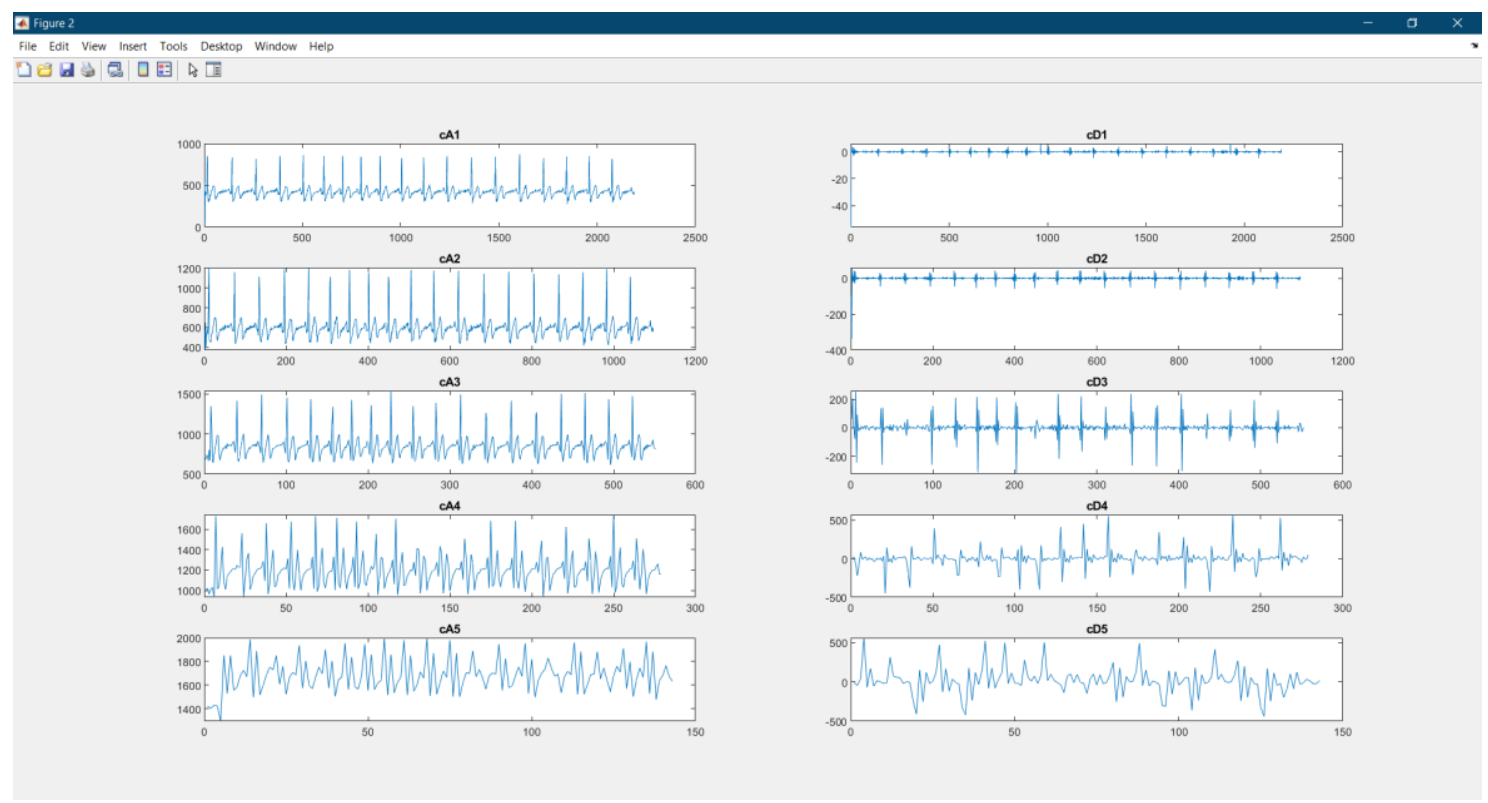

Fonte: Próprio Autor 
A partir dos resultados apresentados na Figura 4 é possível realizar uma análise do sinal de ECG (sinal utilizado para fins de teste) em diferentes bandas de frequência, representadas por componentes de aproximação (cA1, cA2, cA3 e cA4) e componentes de detalhe (cD1, cD2, cD3 e cD4).

\section{CONCLUSÃO E TRABALHOS FUTUROS}

Neste trabalho, demonstramos com êxito o desenvolvimento de um Aplicativo Web Educacional para auxiliar no ensino remoto de processamento digital de sinais. O aplicativo web foi desenvolvido e hospedado com o auxílio das ferramentas App Designer e MATLAB Web App Server do software MATLAB Compiler. O Aplicativo proposto no presente trabalho pode ser acessado e controlado remotamente, através de sua utilização é possível implementar as seguintes técnicas de processamento digital de sinais: filtro passa-alta, filtro passa-baixa, filtro passa- baixa, filtro rejeita-faixa, e decomposição Wavelet multinível utilizando as famílias de funções: Daubechies, Biorthogonal, Coiflets, Symlets, Discrete Meyer, e Reverse Biorthogonal. Também é possível realizar uma análise espectral no domínio da frequência utilizando a FFT (do inglês, Fast Fourier Transform) do sinal analisado. O aplicativo foi testado em situações de acesso remoto externo e local, e em aplicação de um filtro passa (frequência de corte em $64 \mathrm{~Hz}$ ) e um filtro passa faixa (banda passante de 0 à $16 \mathrm{~Hz}$ ), os resultados mostraram o Aplicativo Web Educacional apresentou um bom desempenho durante os testes. Propomos como trabalhos futuros o desenvolvimento de Aplicativos Web Educacional para aplicações em outras áreas da engenharia, com isso, proporcionar uma melhor experiência de ensino remoto para os cursos de engenharia.

\section{Agradecimentos}

Este trabalho foi realizado durante um projeto de pesquisa de mestrado na Universidade da Integração Internacional da Lusofonia Afro-Brasileira. Este trabalho recebeu apoio financeiro da Pró-Reitoria de Pesquisa e Pós-Graduação da UNILAB.

\section{REFERÊNCIAS}

ARRUDA, Eucidio Pimenta. EDUCAÇÃO REMOTA EMERGENCIAL: elementos para políticas públicas na educação brasileira em tempos de Covid-19. Revista EmRede, Porto Alegre, v.7, n.1, p. 257-275, 2020.

BAYILMIS, Cuneyt. Development of a web-based educational interface using MATLAB builder NE with Web Figure for digital modulation techniques. Revista IFAC, Turkey, v.20, n.4, p. 604-610, 2012

DEKEMELE, Kevin; CHEVALIER, Amélie; LOCCUFIER, Mia. ODYSC: A Responsive Educational Web App for Dynamics and Control. Revista IFAC, Tonantzintla, v.51, n.4, p. 310-315, 2018.

FERNANDEZ, R. Sardiñas et al. MATLAB app designer tool to study a microwave photonic filter that integrates analog and digital modulation formats. Revista Optik, Mexico, v.202, 2020. 
JCMWAVE. Matlab Interface-Tutorial. Disponível em:

https://www.docs.jcmwave.com/JCMsuite/html/MatlabInterface/01b770afa0e53824bbb12fca a591f7b2.html?version=4.0.3. Acesso em: 18 de junho de 2020.

MATHWORKS. Matlab Compiler. 2020a. Disponível em:

https://www.mathworks.com/products/compiler.html. Acesso em: 13 de abril de 2020.

MATHWORKS. Matlab App Designer. 2020b. Disponível em:

https://www.mathworks.com/products/matlab/app-designer.html. Acesso em: 22 de abril de 2020.

MATHWORKS. Matlab Web App Server. 2020c. Disponível em:

https://www.mathworks.com/products/matlab-web-app-server.html. Acesso em: 11 de maio de 2020.

MATHWORKS. System Requirementes. 2020d. Disponível em:

https://www.mathworks.com/content/dam/mathworks/mathworks-dotcom/support/sysreq/files/system-requirements-release-2020a-windows.pdf. Acesso em: $11 \mathrm{de}$ junho de 2020.

MATHWORKS. Apps with Auto-Reflow. 2020e. Disponível em:

https://www.mathworks.com/help/matlab/creating_guis/apps-with-auto-reflow.html. Acesso em: 03 de julho de 2020.

UNESCO. Education: From Disruption to Recovery. Disponível em:

https://en.unesco.org/covid19/educationresponse. Acesso em: 16 de junho de 2020.

\title{
DEVELOPMENT OF AN EDUCATIONAL WEB APPLICATION TO ASSIST IN REMOTE TEACHING OF DIGITAL SIGNAL PROCESSING
}

\begin{abstract}
The COVID-19 pandemic contributed to the cancellation of face-to-face classes at universities around the world. As a result, educational institutions had to radically remodel their teaching-learning techniques, taking into account the use of remote teaching tools and platforms. One of the main problems faced by public institutions is the digital inclusion of students in situations of economic vulnerability. These students hardly have a computer with software licensed at home to apply concepts of digital signal processing without depending on the laboratory of the educational institution. Based on these premises, the present work presents the development of an educational web application to assist in the remote teaching of digital signal processing, using a smartphone, computer or tablet. The application works online, the student can access remotely through a link. From the results presented, it is possible to observe that the Web application performed well both in remote access and in the application of signal processing techniques.
\end{abstract}

Keywords: Remote Teaching, Web Application, Signal Processing. 